\title{
Reflections on the article "Moving contact lines and dynamic contact angles: a 'litmus test' for mathematical models and some new challenges" by Yulii D. Shikhmurzaev
}

\author{
Eur. Phys. J. Special Topics 229, 1945 (2020), \\ https://doi.org/ 10.1140/epjst/e2020-900236-8
}

Dieter Bothe ${ }^{\mathrm{a}}$

Department of Mathematics, TU Darmstadt, Darmstadt, Germany

Received 6 July 2020 / Accepted 7 July 2020

Published online 14 September 2020

\begin{abstract}
We carefully consider the 'litmus test' proposed by Yulii D. Shikhmurzaev [Y.D. Shikhmurzaev, Eur. Phys. J. Special Topics 229, 1945 (2020)] in the context of the sharp-interface/sharp-contact line model.
\end{abstract}

The interesting paper [1] starts with a concise survey of a large portion of research in the field of wetting, embedding it into a historical overview and briefly revisiting the 'moving contact line problem'. One main observation of this survey part, being a starting point for the 'litmus test' proposed in the title, is the conclusion that most of the models only differ in rather small details and can all be brought into a common framework in which a relationship of the form

$$
\theta_{d}=f\left(U, \theta_{s}, k_{1}, k_{2}, \ldots\right)
$$

is used to prescribe the dynamic contact angle $\theta_{d}$ as a function of the instantaneous contact line speed $U$. In (1), $\theta_{s}$ is the static (equilibrium) contact angle and $k_{1}, k_{2}$, etc. are material constants characterizing the contacting media; see equation (2) in [1]. Relation (1) is part of the standard model of dynamic contact lines which we recall in some detail below, where we simply write $\theta$ instead of $\theta_{d}$.

Because [1] is focused on the sharp-interface concept, as opposed to diffuseinterface or phase field modeling, we also stick to this approach in which the transition zone between different phases is modeled as a surface of zero thickness, i.e. a family of hypersurfaces embedded into $\mathbb{R}^{3}$. The continuum mechanical description of wetting builds on the modeling of two-phase flows, where a flow domain $\Omega \subset \mathbb{R}^{3}$ is devided into bulk phases $\Omega^{ \pm}(t)$ by means of a $C^{1,2}$-family of moving hypersurfaces $\{\Sigma(t)\}_{t \in J}$ such that $\Omega^{+}(t) \cup \Omega^{-}(t) \cup \Sigma(t)$ is a disjoint decomposition of $\Omega$ for all $t \in J=(a, b)$.

\footnotetext{
* These reflections form an enlarged and revised version of our original report on [Y.D. Shikhmurzaev, Eur. Phys. J. Special Topics 229, 1945 (2020)].

a e-mail: bothe@mma.tu-darmstadt.de
} 
The balances of mass and momentum read

$$
\begin{aligned}
& \partial_{t} \rho+\operatorname{div}(\rho v)=0 \quad \text { in } \Omega \backslash \Sigma, \\
& \partial_{t}(\rho v)+\operatorname{div}(\rho v \otimes v-\mathcal{S})=\rho b \quad \text { in } \Omega \backslash \Sigma,
\end{aligned}
$$

where $\rho$ is the mass density, $v$ the velocity, $\mathcal{S}$ the stress tensor and $b$ denotes body forces. At the interface, the transmission conditions

$$
\begin{aligned}
& \llbracket \rho\left(v-v^{\Sigma}\right) \rrbracket \cdot n_{\Sigma}=0 \quad \text { on } \Sigma, \\
& \llbracket \rho v \otimes\left(v-v^{\Sigma}\right)-\mathcal{S} \rrbracket \cdot n_{\Sigma}=\operatorname{div}_{\Sigma} \mathcal{S}^{\Sigma} \quad \text { on } \Sigma
\end{aligned}
$$

are valid, where $v^{\Sigma}$ is the interface velocity, $n_{\Sigma}$ the interface normal field, $\mathcal{S}^{\Sigma}$ denotes the interface stress tensor and $\operatorname{div}_{\Sigma}$ is the surface divergence. Moreover, no-slip between the bulk phases is usually assumed, i.e. the tangential velocities $v_{\|}^{ \pm}$are continuous, hence

$$
\llbracket v_{\|} \rrbracket=0 \quad \text { on } \Sigma \text {. }
$$

Above, the interfacial jump bracket $\llbracket \cdot \rrbracket$ is defined as

$$
\llbracket \psi \rrbracket(t, x):=\lim _{h \rightarrow 0+}\left(\psi\left(t, x+h n_{\Sigma}(t, x)\right)-\psi\left(t, x-h n_{\Sigma}(t, x)\right)\right),
$$

for $t \in J, x \in \Sigma(t)$. Observe that only the speed of normal displacement $V_{\Sigma}:=v^{\Sigma} \cdot n_{\Sigma}$ of $\Sigma(\cdot)$ enters in (4), (5).

To describe dynamic wetting processes, the interface $\Sigma(t)$ is allowed to touch the domain boundary $\partial \Omega$ (the surface of the substrate) in a set of triple-points $\Gamma(t)$, where all three phases (including the solid phase) meet. It is assumed that at every point of $\Gamma$, a well-defined contact line normal $n_{\Gamma}$ exists, pointing outwards from $\Omega^{+}$, being orthogonal to $\Gamma$ and tangential to $\partial \Omega$. In order to allow for a moving contact line at all, the boundary condition for the interface velocity must allow for slip, at least close to $\Gamma(t)$, and the standard model is the Navier slip boundary condition. A final ingredient is the impermeability condition for the substrate, which has a crucial effect as discussed later. Defining the contact angle $\theta \in[0, \pi]$ via

$$
\cos \theta=-n_{\partial \Omega} \cdot n_{\Sigma},
$$

the remaining model equations read as

$$
v \cdot n_{\partial \Omega}=0, \quad v_{\|}+\alpha\left(\mathcal{S} n_{\partial \Omega}\right)_{\|}=0
$$

for the velocity field (in the frame in which the solid substrate is at rest) with some coefficient $\alpha>0$ and

$$
\theta=f(U)
$$

where, as before, $U$ denotes the contact line speed, defined by $U=v^{\Sigma} \cdot n_{\Gamma}$. Here, equation (10) corresponds to equation (1) above, only that we spare the additional parameters. The geometric notations are illustrated in Figure 1. Since this description employs a sharp contact line, we refer to this model as the sharp-interface/sharpcontact line model.

In many cases, one further specializes to the incompressible two-phase NavierStokes equations, which result from (2)-(5) if the bulk stress is modeled as $\mathcal{S}=-p I+$ 


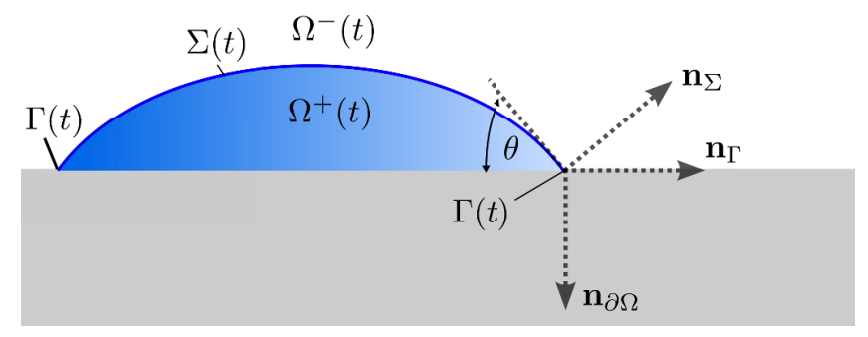

Fig. 1. Illustration of the employed notation.

$\mathcal{S}^{\text {visc }}$, with pressure $p$ and viscous stress $\mathcal{S}^{\text {visc }}=\eta\left(\nabla v+\nabla v^{\top}\right)$, and the interfacial stress tensor as $\mathcal{S}^{\Sigma}=\sigma P_{\Sigma}$ with the surface tension $\sigma$ and the projection $P_{\Sigma}=I-$ $n_{\Sigma} \otimes n_{\Sigma}$ onto the local tangent plane. If, moreover, phase change is excluded and constant densities and viscosities (in the respective phases) as well as a constant surface tension $\sigma$ is assumed, (2)-(6) become

$$
\operatorname{div} v=0 \quad \text { and } \quad \rho\left(\partial_{t} v+v \cdot \nabla v\right)+\nabla p=\eta \Delta v+\rho b \quad \text { in } \Omega \backslash \Sigma,
$$

where $\eta=\eta^{ \pm}$are the constant dynamic viscosities, together with the jump conditions

$$
\llbracket v \rrbracket=0 \quad \text { and } \quad \llbracket p \rrbracket n_{\Sigma}-\llbracket \mathcal{S}^{\mathrm{visc}} \rrbracket \cdot n_{\Sigma}=\sigma \kappa n_{\Sigma} \quad \text { on } \Sigma,
$$

with $\kappa:=\operatorname{div} \Sigma\left(-n_{\Sigma}\right)$ being twice the mean curvature of $\Sigma$. For brevity of notation, we refer to (9)-(12) as the standard model for dynamic contact lines; see, e.g., [2] and the references given there for more details.

The standard model for dynamic contact lines is a semi-empirical model, because empirical information in form of the closure equation (10), respectively (1), is included. Consequently, this model has a limited range of validity in which a quantitative agreement of some desired accuracy holds. It is therefore a relevant research question, where these limits of validity are and how these limits can be identified.

In [1], the author aims at probing existing models for the description of dynamic contact lines via a novel 'litmus test' which considers three prototype wetting experiments: the spreading of a drop on a planar substrate, the rise of a liquid index into a capillary and the wetting of a conical solid. Common to all three cases is the idea to consider the complete wetting process, starting from the moment of initial contact, or even starting from a situation prior to the onset of wetting. Focusing on the planar substrate wetting scenario (see Fig. 3 in [1]), the reasoning then is as follows: if the droplet is brought to the substrate very gently, actually with negligible velocity, there will be an instant of time, say at $t=t_{0}$, where the droplet just touches the planar substrate in a single point, say $x=x_{0}$. This point is then viewed as the starting point of an evolving contact line. Since at time $t=t_{0}$, the tangent plane to the drop at $x_{0}$ coincides with the planar surface of the substrate, a contact angle of $\theta=\pi$ (in radians) is assigned as the initial contact angle. Since the droplet has just touched the substrate in this instant of time and had zero velocity as well as (by implicit assumption) no inner circulation, the contact line speed must be zero at $t=t_{0}$. Hence, the wetting process starts from an initial state which has $(U, \theta)=(0, \pi)$ in the contact angle-versus-speed plane. The droplet then spreads over the substrate and asymptotically reaches an equilibrium state. The latter, being a stationary state, has zero contact line speed and some equilibrium contact angle $\theta_{s} \in(0, \pi)$, the exact value depending on the materials in contact. It is concluded that during the full spreading process, the system state in terms of $(U, \theta)$ runs through a continuous curve, connecting the points $(0, \pi)$ and $\left(0, \theta_{s}\right)$. This implies that for the full interval $\left[0, U_{\max }\right)$ 
of contact line speeds, at least two distinct contact angles $\theta$ need to be assigned to the contact line speed $U$. In other words, the relationship (1), or (10), cannot hold with a well-defined (single-valued) function $f$.

In the second prototype case (see Fig. 5 in [1]), the wetting process starts from $\theta=\pi / 2$ as the angle between the planar liquid surface and the solid wall in the instant of initial contact, otherwise being similar in the line of thought. The third case employs a conical substrate in the thought experiment (see Fig. 6 in [1]), leading to an initial angle which is determined by the opening angle of the solid cone. With these further two arrangements, the same principal idea is used to construct-in theory-a wetting process which starts in a state with $(U, \theta)=\left(0, \theta_{0}\right)$ for a given $\theta_{0} \in[\pi / 2, \pi]$ and develops asymptotically to a state $(U, \theta)=\left(0, \theta_{s}\right)$. Then, for a wetting model to pass the 'litmus test', it is required that the model is able to describe the complete wetting process between these two states. Actually, it is stated in [1] that any model which requires $\theta$ as an input variable fails this test. As already indicated above, the author in [1] concludes that the sharp-interface/sharp-contact line model from above necessarily fails the 'litmus test'.

In order to assess this conclusion, it is helpful to notice that the existence of such conflicting states $(U, \theta)$ already follows if two different states with $U=0$ are possible. But in [1], the latter directly follows from the construction of an initial state $(0, \theta)$ with $\theta \neq \theta_{s}$ from which on the wetting process evolves. At this point, the following two key questions arise:

1. Is the (hypothetical) initial state, coming from one of the scenarios of the above thought experiments, an admissible initial value for the model which is to be assessed by the 'litmus test'?

2. Does this (hypothetical) initial state correspond to a physical state at the onset of a wetting process, i.e., a state which is-in principle-observable in a real experiment?

Question (1) concerns the ability of the sharp-interface/sharp-contact line model for describing, say, the full wetting process of a droplet coming into contact with a planar substrate and then spreading on the substrate, approaching asymptotically an equilibrium state. In order to answer this question for this particular model class, it first has to be clarified in which sense a solution of the sharp-interface/sharp-contact line model has to fulfill the model equations. More precisely, what is the regularity of the solution as a function of time and space and in which sense are the partial differential equations (PDEs), the boundary and initial conditions satisfied. Note that completely different solution concepts are possible for PDEs, depending on whether classical or strong or weak solutions are searched for; cf., e.g., [3] and the references given there for more information concerning the rigorous mathematical analysis of free boundary problems including two-phase flows as well as [4] concerning studies of singularities in hydrodynamics. Now, for our reasoning and throughout this note, we assume sufficient regularity of possible solutions such that the equations make sense in a pointwise manner. In particular, inspecting equations (9)-(12), we assume that the velocity field is jointly continuous in $(t, x)$ and differentiable in $x$, allowing for one-sided limits at the interface $\Sigma(t)$ and these one-sided limits again admit limits at the contact line $\Gamma(t)$. As the solid is modeled as non-permeable, the velocity is tangential to the substrate's surface at $\partial \Omega$. Therefore, the two-phase flow field allows for unique trajectories through any $\left(t_{0}, x_{0}\right) \in J \times \bar{\Omega}$ with $\bar{\Omega}=\Omega \cup \partial \Omega$. In the case without phase change, this has been proven in [2], based on existing results on ordinary differential equations on closed sets. If phase change is allowed, the velocity field becomes discontinuous at the interface. In this setting, the existence of unique trajectories is, mathematically, a highly non-trivial statement. For two-phase flows without contact between $\Sigma$ and $\partial \Omega$, this has been obtained just recently in [5]. To 
sum up, the solution concept underlying our analysis guarantees the existence of a continuous one-to-one flow map

$$
\Psi_{t_{0}}^{t}: \Omega \rightarrow \Omega \quad x_{0} \rightarrow \Psi_{t_{0}}^{t}\left(x_{0}\right):=x\left(t ; t_{0}, x_{0}\right),
$$

where $x\left(\cdot ; t_{0}, x_{0}\right)$ is the unique flow trajectory starting at time $t_{0}$ in the point $x_{0}$ and following the instantaneous local velocity field. The inverse flow map $\Psi_{t}^{t_{0}}$ is also continuous and corresponds to the reversed velocity field $-v$ instead of $v$. Mathematically speaking, $\Psi_{t_{0}}^{t}$ is a homeomorphism on $\Omega$. This condition of continuous motion and deformation has strong implications on the topology of the phases, the interface and the contact line.

Indeed, if one of the phases, representing the droplet, starts as a ball of spherical shape, say, the interface will always be topologically equivalent to a sphere. I.e., the interface $\Sigma(t)$ will be a closed hypersurface without self intersections and without holes. Thus, coming back to the wetting gedankenexperiment above, the spherical droplet can never get perforated, even if it gets into contact with the domain boundary (the substrate).

To overcome this problem, we now take the perforated sphere (i.e., the sphere minus the single point $x_{0}$ at which the sphere touches the surface of the substrate) as the initial interface. The flow field will possibly move and deform the perforated sphere, but the trajectory starting in $x_{0}$ is restricted to move on the surface of the substrate. By an instationary, nonlinear coordinate transform, essentially following the trajectories on the surface of the support, we can assume for simplicity that $x_{0}$ stays fixed. Now suppose, as it should happen in order to describe an evolving wetting process, that a contact line $\Gamma(t)$ is present for $t>t_{0}$ and moves away from $x_{0}$, i.e. the droplet spreads on the substrate. If $x$ is a point inside the contact line $\Gamma(t)$ at a certain time $t>t_{0}$, we follow its trajectory backwards until $t_{0}$. This backward map is given by $\Psi_{t}^{t_{0}}$, hence the backward trajectory ends up in

$$
\Psi_{t}^{t_{0}}(x(t))=\left(\Psi_{t_{0}}^{t}\right)^{-1}\left(x\left(t ; t_{0}, x_{0}\right)\right)=x_{0}
$$

But this means that all points in $\Gamma(t)$ started at $t=t_{0}$ in the single point $x_{0}$, i.e. $\Psi_{t_{0}}^{t}\left(x_{0}\right)=\Gamma(t)$, thus contradicting the unique solvability for the flow trajectories. Consequently, even if we start with a perforated sphere, any regular solution to the sharp-interface/sharp-contact line model-whatsoever-cannot form a contact line from a hole being just a single point. In other words, describing a wetting process via the sharp-interface/sharp-contact line model requires an initial state which already has an initial contact line-however small, but of positive length. Hence, just as the sharpinterface model cannot describe the onset of a new phase, the sharp-interface/sharpcontact line model does not allow for the onset of a new contact line. Notice that this outcome follows solely from the impermeability condition $(9)_{1}$, together with the assumed regularity of the flow field. Hence, this is a purely kinematical property.

So far, we thus found a negative answer to the question whether the sharp interface/sharp contact line model can describe a complete wetting processes, starting from a perforated closed hypersurface or even before the onset of wetting. But this defect is not related to the kind of problem stated in [1], but simply a consequence of the fact that the initial shape of a perforated closed hypersurface, like a sphere without one point, is topologically not equivalent to any shape with a contact line of positive length. Nevertheless, this is an important limitation of the sharp interface/sharp contact line model and the latter, hence, can only describe wetting processes starting from initial values that already contain a contact line $\Gamma_{0}$. Consequently, initial contact angles as well as initial contact line speeds along $\Gamma_{0}$ are required for the model. Now, the decisive point about the proposed 'litmus test' is the question, whether 


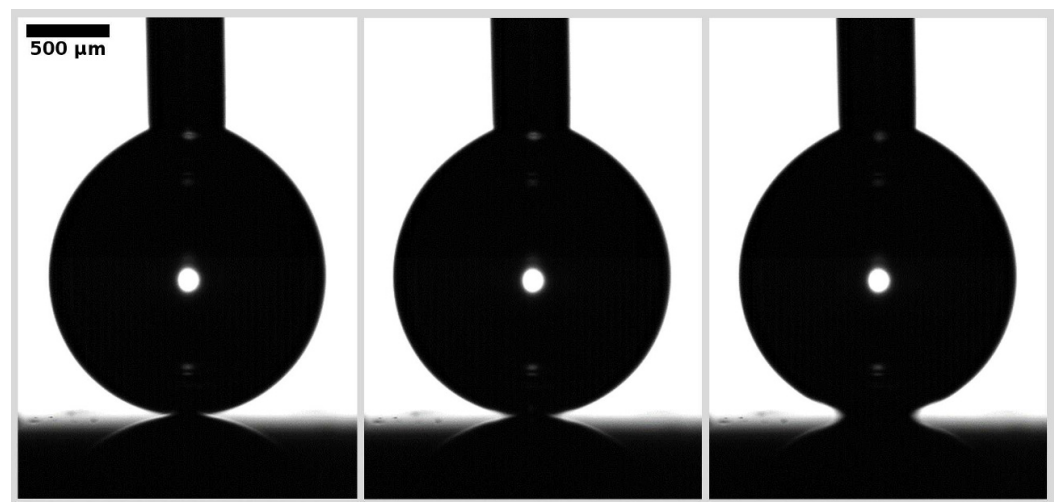

Fig. 2. Initial wetting stage of a water droplet on a silicon wafer. Time interval of $100 \mu \mathrm{s}$ between the successive pictures. Courtesy of S. Hardt, M. Hartmann, TU Darmstadt.

the system's state very shortly after the onset of wetting displays a pair of values $(U, \theta)$ close to $\left(0, \theta_{0}\right)$, where $\theta_{0} \neq \theta_{s}$ is the angle from the hypothetical initial state of the thought experiment. We therefore broaden the above question (2) somewhat by asking

(2)' Is the (hypothetical) initial state (coming from a scenario of the above thought experiment) close to an observable state $(U, \theta)$ for a real wetting process?

The somewhat intuitive conjecture of continuity of $(U(t), \theta(t))$ (say in a rotationally symmetric setup such that only $t$ appears as the independent variable) as a function of $t$ would suggest this to hold true. At a first glance, a purely theoretical approach to answer this question could, possibly, be to solve the equations backwards in time, and to check whether a limit as $t$ approaches the instant of the formation of the contact line exists and what the limiting state is. But this is not even possible in principle, at least not for a viscous fluid, since the backward Navier-Stokes system is ill-posed.

On the other hand, experimental observations indicate that in the early stage of wetting, the contact line speed immediately after the onset of wetting has the highest values. Exemplarily, Figure 2 displays three successive pictures of the early stage of wetting of a water droplet on a silicon wafer with a time interval of $100 \mu$ s between the successive frames. Unfortunately, it is not possible to directly answer the question (2)' with the help of Figure 2, since one can only infer that the onset of wetting took place at some instant between the left and the right frame; let us mention that there is no notable difference between the left picture and frames taken before which are not shown here. Nevertheless, from Figure 2 one can estimate a contact line speed in the order of $1 \mathrm{~m} / \mathrm{s}$ directly after the onset of wetting.

In [6], which is not included in the list of references in [1], a more refined measurement technique with optical access directly to the droplet's foot through a transparent substrate, together with an enhanced high-speed imaging technique, allowing for up to 600.000 frames/s, is applied to study the very early stage of spreading of lowviscosity drops on partially wetting surfaces. As a result, the distance $r$ travelled by the (assumed rotationally symmetric) contact line, as a function of time, scales as $t^{1 / 2}$, i.e.

$$
r \sim t^{1 / 2}
$$

where the instant $t_{0}$ of initial contact is set to 0 . Note that due to the high frame rate, the first picture was taken already $1-2 \mu$ s after the initial contact. So the scaling 


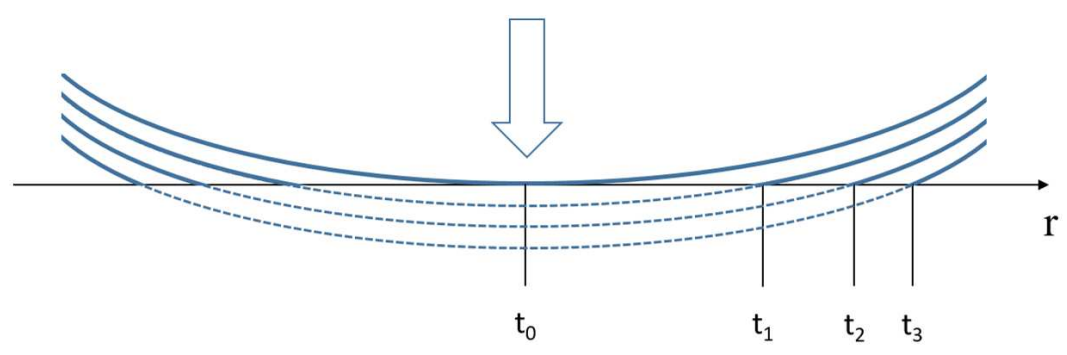

Fig. 3. Conception of initial wetting as a partly kinematic process. Contact line position at successive times $t_{0}, \ldots, t_{3}$.

in (15) holds true at least very early after the onset of wetting. Moreover, this scaling was found to be actually independent of the wettability of the employed substrates (substrates used in [6] are reported to have $\theta_{s} \approx 0, \theta_{s}=65^{\circ}, \theta_{s}=115^{\circ}$ ). Of course this does not rigorously prove that the scaling (15) holds true from the very moment of the onset of wetting, but it strongly indicates that the hypothetical initial value from the thought experiment may be of little or no relevance for the physical process, since the experiment yields a contact line speed $r^{\prime}(t) \sim t^{-1 / 2}$ which diverges as $t \rightarrow 0+$, assuming it to hold for any $t>0$.

This, in turn, raises the question-interesting in itself-whether a contact line speed $U(t) \sim \frac{1}{\sqrt{t}}$ could hold directly from $t>0 \mathrm{on}$. At this point, the following observation, which stems from the scaling behavior (15), seems helpful: Locally around the point $x_{0}$ of initial contact of a sphere, or of a general curved surface (with defined curvature, hence a $C^{2}$-surface) touching the substrate in a single point $x_{0}$, the surface can be approximated as a paraboloid with second order of accuracy. If the contact line moves, starting at $t=0$ in $x_{0}$, say, over a horizontal distance of $r \sim \sqrt{t}$ up to time $t$, the liquid at this distance from $x_{0}$ has moved in vertical direction over a distance proportional-to leading order-to $t$ to reach the substrate. Hence, the observed scaling behaviour of the contact line dynamics would follow if the spreading of the liquid is caused by the liquid 'particles' approaching the substrate from the normal direction as illustrated in Figure 3, even with a (locally) uniform speed. Evidently, this conception of initial wetting as a partly kinematic process requires to give up the impermeability condition $v \cdot n=0$ on $\partial \Omega$. At this point, note that $v \cdot n \neq 0$ follows automatically, if the fluid molecules which actually are in contact to the substrate (thus, wetting it) are separately balanced as its own species of 'adsorbed' molecules, sitting on $\partial \Omega$. This leads to models which account for surface mass balances such as the interface formation model due to Shikhmurzaev [7] or derivatives thereof, such as the model recently proposed in [8]; see also [9] for a mathematical discussion of the latter model.

Coming back to the simplifying kinematic picture of the very early stage of wetting above, it still remains to check if the initially infinite contact line speed would, in principle, be physically possible. Once the contact line is formed, the surface tension force at $\Gamma$ is out of balance and the surface energy stored in the droplets shape can perform the work necessary to squeeze the gas phase out of the contact line region. Until each time $t>0$, the volume below the paraboloid, intersected with a vertical cylinder of radius $r(t)$ has to be squeezed to the outside. To simplify this qualitative reasoning, we assume that in the interval $[t, t+\Delta t]$, a cylindrical barrel of inner radius $r(t)$, thickness $r^{\prime}(t) \Delta t$ and height $r(t)^{2}$ needs to be accelerated to reach a speed of $r^{\prime}(t)$ in order to precede the moving contact line. This corresponds to a change of 
momentum at a rate which is proportional to

$$
r(t)^{3} r^{\prime}(t)^{2} \sim \sqrt{t}^{3}\left(\frac{1}{\sqrt{t}}\right)^{2} \sim \sqrt{t}
$$

i.e. a force of precisely this scaling is required to fulfil Newton's second law. On the other hand, the surface tension force $F_{\sigma}$ acting on the contact line is proportional to the length of $\Gamma$, i.e.

$$
F_{\sigma}(t) \sim|\Gamma(t)|=2 \pi r(t) \sim \sqrt{t}
$$

hence $F_{\sigma}$ is exactly of the right scaling in order to explain the observed relationship. Let us note in passing that if a scaling of $r \sim t^{\beta}$ for some $\beta>0$ is assumed, the line of arguments from above yields $5 \beta-2=0.5$ as a necessary condition, hence $\beta=0.5$. Since (16) and (17) hold for all $t$ backward in time up to the onset of wetting, this gives additional evidence for the scaling behavior (15) to hold for all $t>0$. More general, it supports the conjecture that wetting does not necessarily start with small contact line speed. Therefore, the answer to question (2)' from above very likely is in the negative.

The author of [1] apparently expected this kind of objection, since he writes that "a reader without a background in mathematical modelling but keen on protecting the approach outlined in (Sect. 2 of [1]) is bound to suggest that as a drop touches a substrate at what in macroscopic terms is a point, there is not yet any contact line as such and hence one should not attribute any speed to it nor extract a contactangle value from the initial configuration". The text resumes, offering as a remedy: "Although this kind of objection is removed by a simple continuity argument, this category of readers is best addressed by referring them to Figure 4 sketching a drop deposited onto and spreading over a profiled substrate. In this setting, the base of the drop is finite and a finite-length contact line is present from the start. The rest of the argument is the same as for the flow shown in Figure 3." The mathematical reasoning above shows that such a "simple continuity argument" simply cannot exist. Regarding the thought experiment in which the spherical droplet wets a profiled substrate, say an inverted conical shape with the corner rounded off to form a spherical cap of some opening angle less than $\pi / 2$ and of the same radius as the droplet in order to create a positive area of initial contact, note that this gedankenexperiment would require that the gas film drains without any deformation of the interface and that the initial wetting simultaneously happens on the full spherical cap region, while the onset of wetting requires a nucleation-like process to trigger the topological change. Moreover, in this thought experiment, the surface energy of the droplet surface contacting at once the spherical cap would be assumed to just disappear without any effect. In our opinion, this hypothetical situation is also not physically plausible.

To conclude, the 'litmus test' under consideration is not appropriate to falsify the standard model for dynamics contact lines. On the other hand, the sharp-interface/sharp-contact line model cannot predict the onset of wetting, at least not with regular solutions under the condition $v \cdot n=0$ at the surface of the substrate.

Let us finally note that the latter two assumptions also have strong implications on the asymptotic behavior: Indeed, as proven in [2], if a regular solution of the standard model for dynamic contact lines, i.e. of the model (9)-(12), asymptotically reaches the equilibrium state, the second law of thermodynamics cannot be fulfilled at all times during the evolution! Put the other way around, the entropy inequality imposes an additional condition on $f$ from (10) and for such $f$, it has been shown in [2] that a regular solution of the standard model cannot reach the equilibrium contact angle. Such a statement cannot be obtained without sufficient regularity of solutions. 
Consequently, and this might be the more important conclusion, the impermeability condition-just as the no-slip condition-should be seriously questioned in the mathematical modeling of dynamic contact lines.

It is our strong believe that further progress in this interesting and relevant research area can be achieved via interdisciplinary efforts.

Open access funding provided by Projekt DEAL. The author expresses his thanks to Mathis Fricke for fruitful discussions on the sharp-interface/sharp-contact line model and acknowledges financial support by the German Research Foundation (DFG) within the Collaborative Research Centre 1194 Interaction of Transport and Wetting Processes, Project-ID 265191195, sub-project B01.

Open Access This is an open access article distributed under the terms of the Creative Commons Attribution License (http://creativecommons.org/licenses/by/4.0), which permits unrestricted use, distribution, and reproduction in any medium, provided the original work is properly cited.

Publisher's Note The EPJ Publishers remain neutral with regard to jurisdictional claims in published maps and institutional affiliations.

\section{References}

1. Y.D. Shikhmurzaev, Eur. Phys. J. Special Topics 229, 1945 (2020)

2. M. Fricke, M. Köhne, D. Bothe, Physica D 394, 26 (2019)

3. J. Prüss, G. Simonett, Moving interfaces and quasilinear parabolic problems. Monographs in Mathematics (Birkhäuser, 2016)

4. J. Eggers, M. Fontelos, Singularities: formation, structure, and propagation, Cambridge Texts in Applied Mathematics (Cambridge University Press, Cambridge, 2015)

5. D. Bothe, Nonlinearity (2020, in press), doi: 10.1088/1361-6544/ab987d

6. K.G. Winkels, J.H. Weijs, A. Eddi, J.H. Snoeijer, Phys. Rev. E 85, 055301(R) (2012)

7. Y.D. Shikhmurzaev, Int. J. Multiph. Flow 19, 589 (1993)

8. A.V. Lukyanov, T. Pryer, Langmuir 33, 8582 (2017)

9. M. Fricke, D. Bothe, Eur. Phys. J. Special Topics 229, (2020) 